\title{
Recent advances in multi-dimensional metasurfaces holographic technologies
}

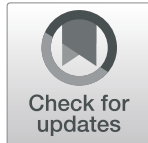

Ruizhe Zhao, Lingling Huang ${ }^{*}$ and Yongtian Wang ${ }^{*}$

\author{
* Correspondence: huanglingling@ \\ bit.edu.cn; wyt@bit.edu.cn \\ School of Optics and Photonics, \\ Beijing Institute of Technology, \\ Beijing 100081, China
}

\begin{abstract}
Holography has attracted tremendous interest due to its capability of storing both the amplitude and phase of light field and reproducing vivid three-dimensional scenes. However, the large pixel size, low resolution, small field-of-view (FOV) and limited space-bandwidth of traditional spatial light modulator (SLM) devices restrict the possibility of improving the quality of reconstructed images. With the development of nanofabrication technologies, metasurfaces have shown great potential in manipulating the amplitude, phase, polarization, frequency or simultaneously multiple parameters of output light in ultrashort distance with subwavelength resolution by tailoring the scattering behaviour of consisted nanostructures. Such flexibilities make metasurface a promising candidate for holographic related applications. Here, we review recent progresses in the field of metasurface holography. From the perspective of the fundamental properties of light, we classify the metasurface holography into several categories such as phaseonly holography, amplitude-only holography, complex amplitude holography and so on. Then, we introduce the corresponding working principles and design strategies. Meanwhile, some emerging types of metasurface holography such as tunable holography, nonlinear holography, Janus (or directional related) and bilayer metasurfaces holography are also discussed. At last, we make our outlook on metasurface holography and discuss the challenges we may face in the future.
\end{abstract}

Keywords: Metasurface, Holography, Wavefront modulation

\section{Introduction}

Holography invented by Gabor provides a promising technology for restoring and reconstructing the full wave information of object targets. The holograms are generated by the interference between the reference beam and objective beam. When the reference beam illuminating on the hologram, the reconstructed images can be observed on the predefined position [1]. While in 1966, Lohmann and Brown proposed and fabricated the computer generated holography for spatial filter for the first time by introducing the sampling law and detour phase coding method [2]. Holographic related technologies have been applied in many areas such as display, imaging, optical data storage, microscopy and metrology [3]. Fukushima et al. realized the first real-time hologram by using an opticallyaddressable ferroelectric liquid-crystal spatial light modulator in 1990 [4]. A holographic

(c) The Author(s). 2020 Open Access This article is licensed under a Creative Commons Attribution 4.0 International License, which permits use, sharing, adaptation, distribution and reproduction in any medium or format, as long as you give appropriate credit to the original author(s) and the source, provide a link to the Creative Commons licence, and indicate if changes were made. The images or other third party material in this article are included in the article's Creative Commons licence, unless indicated otherwise in a credit line to the material. If material is not included in the article's Creative Commons licence and your intended use is not permitted by statutory regulation or exceeds the permitted use, you will need to obtain permission directly from the copyright holder. To view a copy of this licence, visit http://creativecommons.org/licenses/by/4.0/. 
image can be obtained under readout beam illuminating. However, the resolution of SLM is nearly a few microns, which degrade the quality of reconstructed image and lead to many undesired diffraction orders. Moreover, other defects such as the narrow bandwidth, small FOV and limited space-bandwidth product prohibit holographic related technologies to be applied in practical applications [5-9].

With the development of nanofabrication technologies, metasurfaces consisting of arrays of plasmonic or dielectric nanoantennas have attracted enormous interest due to their ability to arbitrarily tailor the fundamental properties of the electromagnetic wavefront such as amplitude [10-12], phase [13-17], frequency [18, 19], polarization [20-22] and orbital angular momentum (OAM) [23-25] within ultrashort distance. Meanwhile, simultaneously multiple parameters modulation methods have also been demonstrated [26-30], which make metasurface become a potential substitute for the traditional bulky optical elements. Metasurfaces have successfully achieved many novel applications of beam shaping [31-34], metalens [35-39], circular dichroism [40, 41], nonlinear optics $[42,43]$, color printing $[44,45]$ and holography [46-49] . Among these useful applications, metasurface holography is very attractive due to its superior performance over traditional holography. The current state-of-the-art holographic devices such as SLM can only provide either phase or amplitude modulation of light, while the complex amplitude modulation is necessarily required to completely reconstruct full wave information of the wavefront of light in holography. However, methods based on metasurfaces can overcome this obstacle by utilizing various kinds of wavefront manipulating mechanisms. Meanwhile, due to the subwavelength period of metasurfaces the reconstructed images of metasurfaces holography can achieve high resolution with good quality and only the desired diffraction order can be obtained. Besides, the working bandwidth, FOV and spacebandwidth product can also be increased by utilizing delicately designed metasurfaces. Therefore, metasurfaces have been considered as promising devices for achieving applications such as display, imaging, encryption and so on [50].

In this paper, we review recent progresses in metasurface holography. We classify current metasurface holography into non-multiplexed and multiplexed (multi-dimensions wavefront modulations) metasurface holography. We introduce phase-only, amplitude-only and complex amplitude holography in the first part. Then, we investigate several holographic multiplexing strategies such as color holography, polarizationmultiplexed holography (vectorial holography), angle-multiplexed holography, OAMmultiplexed holography as well as spectral- and spatial-multiplexed metasurface. Meanwhile, other types of metasurface holography such as tunable holography, nonlinear holography, Janus (or directional related) and bilayer metasurfaces holography are also discussed. Finally, an outlook for metasurface holography is discussed.

\section{Non-multiplexed metasurface holography}

In this section, we discuss recent advances in phase-only holography, amplitude-only holography and complex amplitude holography based on metasurfaces.

\section{Phase-only holography}

Traditional optical elements such as lenses and waveplates manipulate the wavefront of output light rely on controlling the optical path of light. In this way, phase and polarization 
change can be accumulated when the light propagating through these optical elements. However, the thicknesses of these optical elements are always much larger than the wavelength of incident light which prohibit them from being used in compact and integrated optical systems. Metasurface provide a platform for manipulating the wavefront of output light within ultrashort distance in subwavelength resolution [51-53]. By utilizing the principles of dynamic phase or geometric phase, many methods have been proposed for trailoring the phase of output light in the range of 0 to $2 \pi$ based on plasmonic or dielectric metasurfaces in recent years [15-17].

Geometric metasurfaces provide a simple method for fully controlling the phase of output light. The abrupt phase is dispersionless and solely depends on the orientation angle of the antennas $[15,16]$. Huang et al. [46] demonstrated a metasurface hologram which can encode the desired phase profile in the opposite handedness polarization state of the output light based on geometric phase principle and achieve the reconstruction of 3D image with high resolution, large FOV, free of multiple-order diffractions and twin images. Due to the intrinsic ohmic dissipation of plasmonic material, the diffraction efficiency of proposed hologram remained too low at visible wavelengths for practical purposes. To solve this problem, Zheng et al. [47] reported the design and realization of a reflective geometric metasurface hologram reaching diffraction efficiency of $80 \%$ at $825 \mathrm{~nm}$ and a broad bandwidth between $630 \mathrm{~nm}$ and $1050 \mathrm{~nm}$. By taking advantage of the accurate phase control with geometric metasurfaces and the Fabry-Pérot cavity, the diffraction efficiency of metasurface hologram can be enhanced dramatically without the need of a complicated fabrication process.

While the above proposed geometric metasurfaces are only suitable for the circularly polarized light, different mechanisms have been proposed for linearly polarized light or polarization insensitive illumination and improving the diffraction efficiency $[54,55]$. By employing the concept of the multi-resonant response based on the generalized Huygens' principle, a dielectric metasurface composed of $\mathrm{Si}$ nanodisks with different radius was proposed to achieve high resolution grayscale images encoding [54]. Such Huygens' metasurface can achieve uniform transmission while the phase modulation can cover $2 \pi$, by obtaining the equivalent strength of the spectrally overlapping of electric and magnetic dipole resonances. And the diffraction efficiency of the dielectric metasurface can reach over $90 \%$ at a wavelength of $1600 \mathrm{~nm}$. The all-dielectric geometric metasurfaces opens another possibility for achieving high efficiency holograms in transmission mode. Meanwhile, dielectric metasurfaces with birefringent property can be designed as half-wave plates for achieving high efficiency circular polarization conversion by optimizing the geometry parameters of cross sections. This is because light propagating along the long and short axis of meta-atom can result in different effective refractive index, which lead to different phase delays [56]. Furthermore, by employing propagation phase together with geometric phase, more phase control flexibilities can be achieved [26, 27]. Dielectric metasurfaces composed of arrays of $\mathrm{Si}$ or $\mathrm{TiO}_{2}$ nanorods with spatially-varying orientation angles were demonstrated for achieving high quality and fidelity reconstructed images in visible or near-infrared region with high efficiency $[26,27,55]$. 


\section{Amplitude-only holography}

The phase profiles of most phase only metasurface holograms are generated by using Gerchberg-Saxton (GS) or point source algorithm which the amplitude distributions are usually set uniform. However, as another important design freedom, the amplitude can also be applied to the reconstruction of the target image, especially for those cases where the phase information cannot be employed. Meanwhile, the designed nanostructures for achieving amplitude modulation are relative simple (usually composed of nanoholes arrays with same sizes) and the qualities of reconstructed images are acceptable.

For amplitude-only metasurface holography, the transmitted or reflective amplitude of each unit cell can be quantitatively divided to different levels. Among all the types of amplitude holograms, binary amplitude hologram is the most common case which the amplitude distribution is composed of the values 0 and 1 . Although only two amplitude levels are contained in binary amplitude hologram, the one bit digital coding of 0 and 1 can also reproduce holographic images with satisfactory quality. By utilizing the scattering of arrays of vertically aligned multiwalled carbon nanotubes, the demonstrated binary amplitude holograms can achieve low noise and high resolution images with wide FOV [57]. However, the twin images can't be avoided in the reconstruction. To solve this problem, Huang et al. [11] realized a uniform, twin-image free and high efficiency binary hologram based on analysing the diffracted field from a huge number of subwavelength photon sieves and optimizing with a genetic search algorithm. Furthermore, Walther et al. [58] explicitly used nanoapertures within the metal film of different sizes to tune the transmission coefficients and achieved binary amplitude holography at two wavelengths. Recently, $\mathrm{Xu}$ et al. [10] investigated the correlation between different photon sieves and proposed an amplitude-only holographic strategy which can generate two different binary amplitude holograms as shown in Fig. 1a. By developing a modified GS algorithm, two binary amplitude distributions (an upper set $A$ and a subset $B$ with rigorous mathematical relationship $A \subset B$ ) were generated. The subset can be considered as shutting/switching a part of 1 to 0 from the upper set, and the two correlated amplitude distributions can reconstruct two completely different holographic images in the Fourier plane.

\section{Complex amplitude holography}

In order to improve the quality of holographic images and completely reconstruct the images without losing any information, the amplitude and phase modulations are both necessary. Compared to traditional SLMs which can only provide the phase or amplitude modulation, some methods have recently been proposed for realizing complex amplitude modulation based on metasurfaces [59-62].

For complex amplitude modulation, some methods have been proposed based on the resonance properties to control the phase and amplitude of output light [61], or by combining the resonance properties together with geometric phase principle [62]. Furthermore, the superposition of two independent modes of the PB phase provided by the meta-atoms within one unit cell can result in arbitrary complex amplitude modulation through elaborately choosing the orientation angles of the consist meta-atoms [59]. 


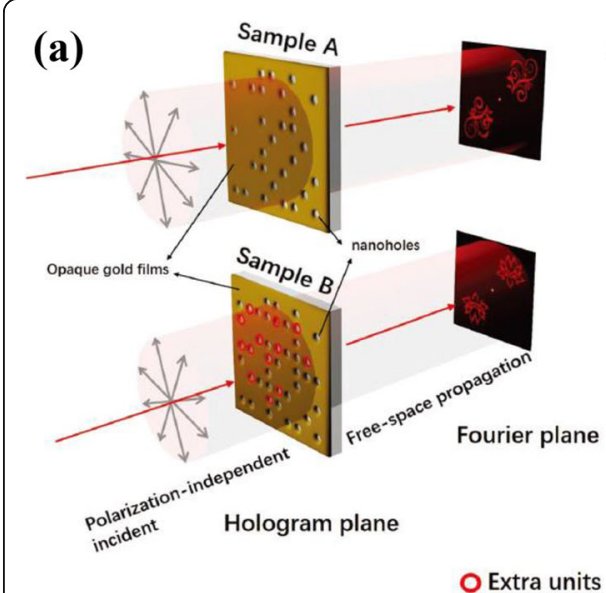

(b)

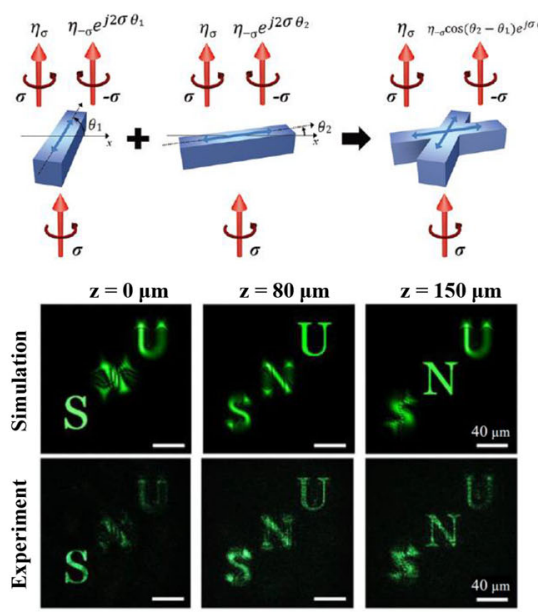

(c)
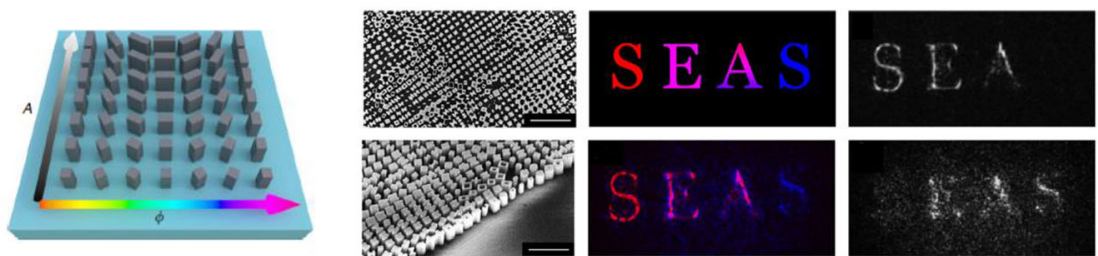

Fig. 1 Amplitude-only holography and complex amplitude holography based on metasurfaces. a Schematic illustrations of demonstrated photon sieves. Two completely different holographic images can be reconstructed through quantitatively correlated amplitude modulations [10]. b Complex amplitude holography based on dielectric metasurfaces composed of X-shaped meta-atoms. The principle is shown on the top panel. The simulated and experimental results of proposed complex amplitude hologram are shown on the bottom panel [59]. c Dielectric metasurfaces composed of amorphous silicon (a-Si) nanofins with different sizes and orientation angles for complete and independent control of complex amplitude under circular polarization illumination. The scanning electron micrograph (SEM) images of fabricated metasurfaces are shown on the middle. Complex amplitude modulation can be obtained at two different frequencies and result in the reconstruction of holographic images [60]

V-shape nanoantennas and C-shape split-ring resonators (CSRRs) are commonly adopted structures to achieve complex amplitude modulation. By utilizing the symmetric and anti-symmetric resonance mode of $\mathrm{V}$-shape nanoantennas, two-level amplitude and eight-level phase modulation are realized and can be used to achieve the reconstruction of high resolution and low-noise images in the visible range [61]. To increase the amplitude levels, CSRRs are chosen as the basic unit to achieve five-level amplitude and eight-level phase modulation at terahertz frequencies [62]. The phase and amplitude of the output orthogonally-polarized linear light can be simultaneously and independently controlled by changing the size (radius $r$, split angle $\alpha$ ) and orientation angle $\theta$ of CSRRS based on symmetric and anti-symmetric resonance mode together with geometric phase principle.

In order to achieve complex amplitude modulation in visible region with high efficiency, Lee et al. [59] demonstrated a novel dielectric metasurface composed of Xshaped meta-atoms that exhibit the ability to control the amplitude and phase of visible light independently with subwavelength spatial resolution. As shown in Fig. 1b, two arms of X-shaped meta-atom can possess two independent modes for the PB phase that controlled independently by their orientation angles $\theta_{1}$ and $\theta_{2}$. 


$$
\begin{gathered}
E_{\text {cross }} \propto e^{j 2 \sigma \theta_{2}}+e^{j 2 \sigma \theta_{1}}=\left(e^{j \sigma\left(\theta_{2}-\theta_{1}\right)}+e^{-j \sigma\left(\theta_{2}-\theta_{1}\right)}\right) e^{j \sigma\left(\theta_{1}+\theta_{2}\right)} \\
=2 \cos \left(\theta_{2}-\theta_{1}\right) e^{j \sigma\left(\theta_{1}+\theta_{2}\right)}
\end{gathered}
$$

The cross-polarized component of transmitted light is expressed by Eq. (1). The amplitude and phase of arbitrary complex electromagnetic field are only determined by $\theta_{1}$ and $\theta_{2}$. This independent, continuous and broadband complex amplitude modulation successfully provides vivid complex amplitude holograms with much higher signal-tonoise ratio (SNR) compared to the phase-only holograms in the entire visible region. Recently, Overvig et al. [60] proposed an approach, that used meta-atoms with a varying degree of form birefringence and orientation angles to create high efficiency dielectric metasurfaces that control both the amplitude and phase at one or two frequencies. The modulation mechanism is shown in Fig. 1c, the amplitude is controlled by varying the cross-polarization conversion efficiency of circularly polarized light via tailoring the size of birefringent meta-atoms, while the phase is a sum of the propagation phase and the geometric phase. From the experimental results we can find the quality of reconstructed images of complex amplitude metasurfaces is superior over phase-only metasurfaces holography. Meanwhile, complex amplitude metasurfaces enable a few features not attainable in phase-only holography such as creating artifact free 2D holographic images, controlling the surface textures of 3D holographic objects, encoding phase and amplitude profiles separately at the object plane and obtaining different intensity profiles at the metasurface and object planes separately.

\section{Multiplexed metasurface holography}

Multiplexing techniques are highly desired to optimize the tremendous information capability and improve the space-bandwidth product of metasurface holograms by increasing the design freedoms. And the fundamental properties of light such as amplitude, phase, frequency, polarization, OAM, together with simultaneously control of several parameters, can be utilized as the design freedoms. Therefore, in this section we introduce the recent developments in multiplexed (multi-dimensions) metasurface holography including color holography, polarization-multiplexed holography (vectorial holography), angle-multiplexed holography, OAM-multiplexed holography as well as spectral- and spatial-multiplexed metasurface.

\section{Color holography}

Realizing the independent phase or complex amplitude modulation in different wavelengths in order to achieve color holography is very necessary for practical applications such as imaging and display.

Most of the current color holography strategies adopted spatial multiplexing schemes to obtain different responses at different wavelengths [63, 64]. Other schemes utilized multiwavelength GS algorithm to generate the hologram and reconstruct the color images at designed plane [65]. For example, an aluminum plasmonic metasurface that can realize twolevel phase modulation for each primary color is demonstrated based on spatial multiplexing schemes [63]. The subpixel is composed of aluminum nanorods with same sizes, but the subpixels from different pixels can have different sizes yield either 0 or $\pi$ phase shifts for a given color and achieve color holography. However, the spatial multiplexing schemes degrade the resolution of reconstructed images. Meanwhile, the narrow bandwidth of the resonances of 
the aluminum rods make the efficiency of proposed metasurfaces can reach only around $0.3 \%$. For achieving high efficiency color holography, Wang et al. [64] proposed a silicon metasurface formed by three kinds of nanoblocks multiplexed in a subwavelength unit to constitute a meta-atom which was capable of manipulating the wavefront of the three primary colors simultaneously. The nanoblocks can work as half waveplates at the non-overlapping discrete wavelengths by optimizing their geometric parameters due to birefringence phenomenon and fulfill full phase control for the three primary colors with high efficiency independently.

For improving the image quality of color holography and reducing the crosstalk among different colors in broadband, Li et al. [66] developed a method based on off-axis illumination with a single type of plasmonic nanoaperture. This method leads to a remarkable image quality with high SNR, and achieve large color gamut, but with low efficiency. While, once the metasurface has been fabricated, it becomes very challenging to tune the color of the reconstructed images. Wang et al. [67] demonstrated a spin-controlled color tunable hologram based on Si metasurface as shown in Fig. 2a. By utilizing spatial multiplexing schemes and geometric phase principle, the designed holographic images for each primary color are encoded in the RCP or LCP component of output light. For any other incident polarization which can be decomposed to the RCP and LCP components with different intensity ratio, the intensities of the twin images varied according to the varying intensity ratio which result in tunable colors by color mixing.

Recently, a multitasked metasurface with non-interleaved single-size Si nanobrick arrays was proposed for encoding 6-bit information for color holography [65]. The schematic diagram of the mechanism is presented in Fig. $2 \mathrm{~b}$. The red $\hat{R}$, green $\hat{G}$, and blue $\hat{B}$ wavelengths with two different spins are used to represent six fundamental bases $\left(\hat{\mathrm{R}}_{\mathrm{RCP}}, \hat{\mathrm{G}}_{\mathrm{RCP}}\right.$, $\hat{\mathrm{B}}_{\mathrm{RCP}}, \hat{\mathrm{R}}_{\mathrm{LCP}}, \hat{\mathrm{G}}_{\mathrm{LCP}}$, and $\hat{\mathrm{B}}_{\mathrm{LCP}}$ ). Based on the dispersion relationship of propagation, the multiwavelength Gerchberg-Saxton (MWGS) algorithm is adopted to generate the desired phase profiles which can be encoded to the metasurfaces according to geometric phase. The reconstruction of $2^{6}-1$ spin and wavelength dependent holographic images is successfully achieved by manipulating six fundamental bases of the input beams at carefully

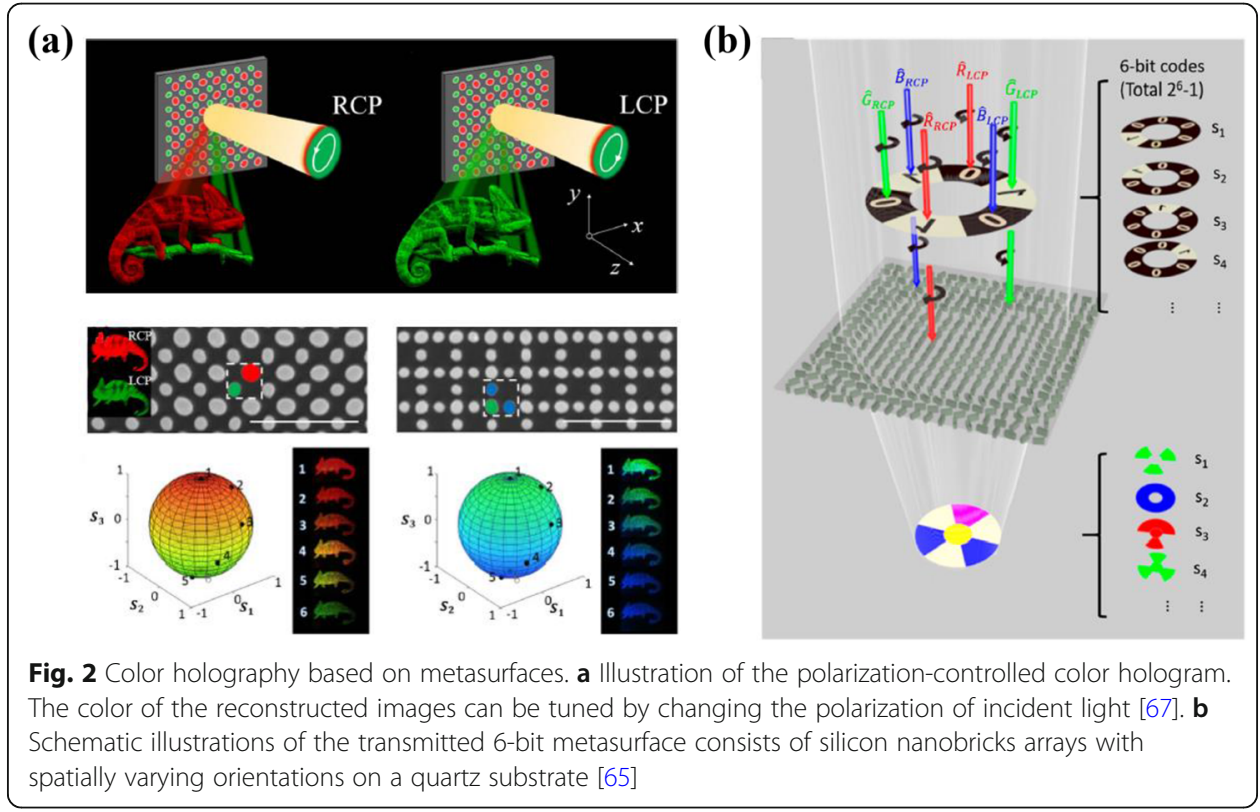


designed observed plane. Other color holography scheme has been achieved by utilizing multiple polarization channels. A non-interleaved $\mathrm{TiO}_{2}$ metasurface hologram is demonstrated with capabilities of encoding three distinct phase profiles in different polarization channels. Combining three polarization channels with three primary color channels, a full-color hologram with nearly no crosstalk is realized [68].

\section{Polarization-multiplexed holography}

Compared to natural materials which are usually polarization insensitive or only possess weak polarization rotation abilities, the metasurfaces offer the flexibility to completely alter the polarization state of the interacting light based on the artificially tailored meta-atoms that make them become suitable for achieving polarizationmultiplexed holography $[69,70]$.

Anisotropic structures are widely adopted to achieve polarization-multiplexed holography due to birefringence phenomenon. Montelongo et al. [71] proved theoretically and experimentally the concept of polarization holography by utilizing the radiation emitted by plasmonic nanoantennas. The selectively activation of the nanoantenna emission can be controlled by tuning the incident polarization. Two sets of independent transverse nanoantennas in the same plane are merged and constitute the final switchable metasurface which is capable of reconstructing two polarization controlled high resolution images over a wide FOV in the far field. Another kind of helicitymultiplexed metasurface hologram was achieved based on geometric phase principle due to its helicity switchable property [72].

Dielectric metasurfaces based on high-contrast dielectric elliptical nanoposts were demonstrated for simultaneously controlling the polarization and phase of the output light with subwavelength spatial resolution by designing both the eigenvectors together with rotation matrix of the dielectric resonators [26]. The general relation between the electric field of the input and output lights at each pixel can be expressed using the Jones matrix. For metasurfaces with high transmission, the input light $\left(E_{i n}\right)$ can be mapped to arbitrary desired output light $\left(E_{\text {out }}\right)$ using a symmetric and unitary Jones matrix if the polarization dependent phase shift $\left(\phi_{x}, \phi_{y}\right)$ and the azimuthal angle $\theta$ can be chosen freely. Based on proposed wavefront modulation method, a polarization-switchable phase hologram that generates two distinct patterns for $x$ - and $y$-polarized light is achieved with diffraction efficiency over $80 \%$ at the near-infrared wavelength of $915 \mathrm{~nm}$. Furthermore, Mueller et al. [27] extended this method and realized encoding two independent and arbitrary phase profiles on any pair of orthogonal states of polarization-linear, circular, or elliptical with birefringent metasurfaces by combining both propagation and geometric phases.

Although the method proposed by Mueller et al. breaks the limitation of the helicity multiplexed metasurface that can only obtain the twin images when switching the helicity of input circularly polarized light, many efforts have still been made in the areas of polarization multiplexed holography and vectorial holography in recent years. Deng et al. [28] demonstrated a new concept of vectorial holography based on diatomic metasurfaces consisting of metamolecules formed by two orthogonal meta-atoms arranged as a periodic array with a periodicity $p_{0}$ as shown in Fig. 3a. Two orthogonal 


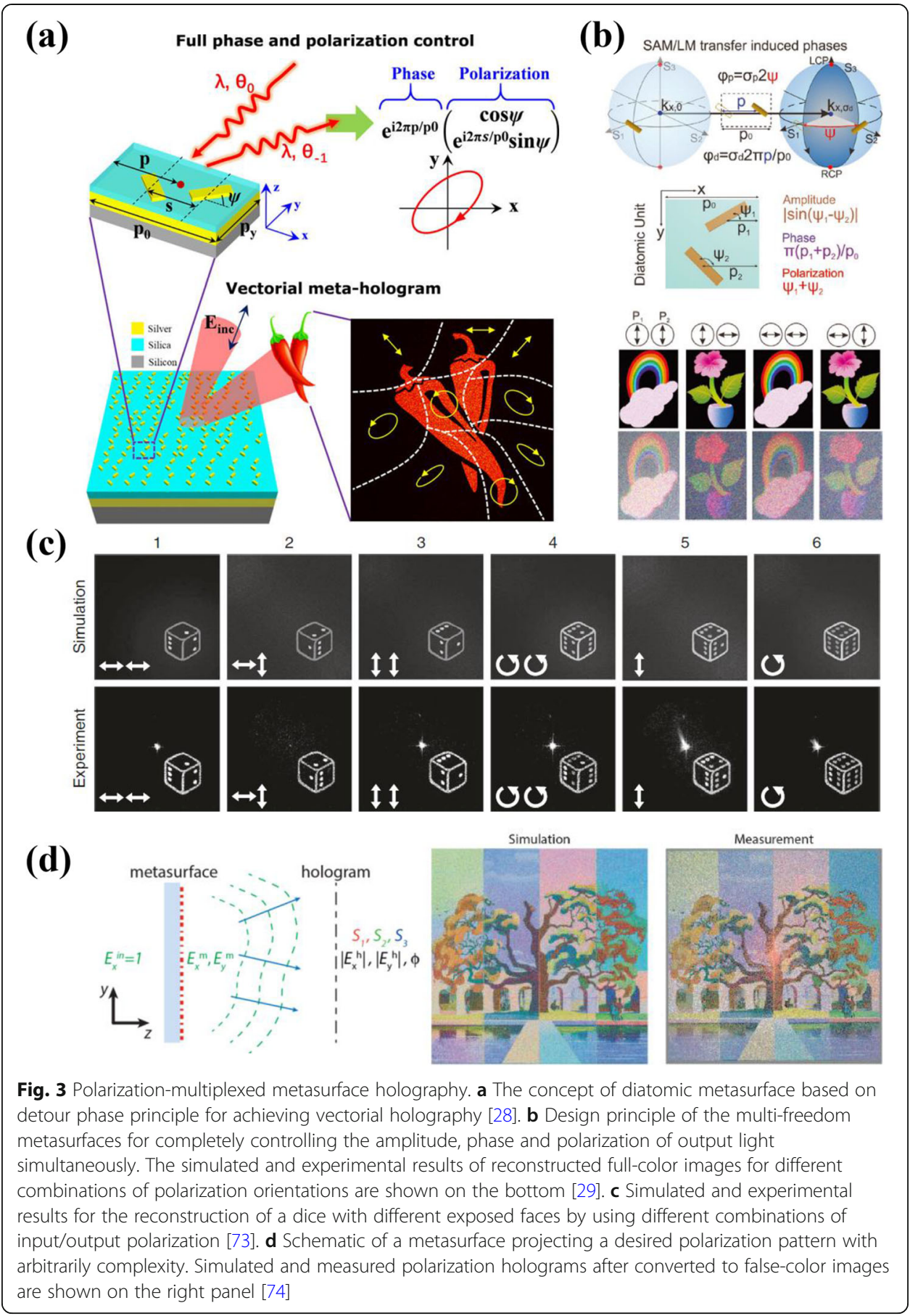

polarization components can be generated by the anisotropic meta-atoms for achieving fully polarization control. By considering the optical path difference and the momentum conservation under TE polarized light illuminating, the Jones vector of the -1 st scattered light from the designed diatomic metasurfaces can be expressed by Eq. (2) where $C$ is the coupling constant, $s$ is the local displacement between orthogonal metaatoms, $p$ is the global displacement of adjacent meta-molecules and $\Psi$ indicates the orientation angle of meta-atoms. 


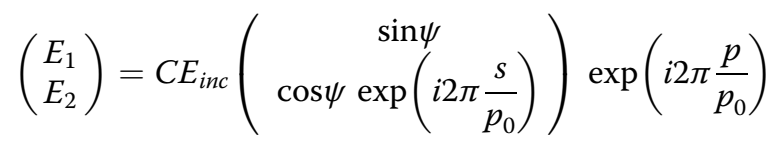

We can find that the arbitrary phase and polarization modulation can be achieved by tailoring the displacements and orientation angles of identical meta-atoms which is robust against both incident angles and wavelengths. Therefore, broadband vectorial holographic images with spatially varying polarization states and dual-way polarization switching functionalities have been successfully demonstrated based on this feature. Furthermore, by combination the dispersionless geometric phase and detour phase (proportional to the displacement between adjacent meta-elements), Deng et al. [29] demonstrated a multi-freedom metasurface that can simultaneously and independently modulate phase, polarization, and amplitude. Meanwhile, wavelength multiplexing is further realized by a k-space engineering technique as shown in Fig. 3b. Inspired from the pioneer works of completely controlling the phase and polarization of output light simultaneously, Zhao et al. [73] provided another polarization multiplexing scheme to further expand the polarization channels and enhance multiplexing ability. Multiple independent target phase profiles with quantified phase relations that can process significantly different information in different polarization states are realized within a single metasurface. By selecting the suitable input/output polarization combinations, 12 channels in total with seven different combinations can be reconstructed with high quality and fidelity as shown in Fig. 3c.

Meanwhile, vectorial holpgraphy can also be realized by utilizing phase-only metasurfaces [75] or geometric-phase metasurfacces [76]. Equivalent electromagnetic model and gradient-based nonlinear optimization are adopted to achieve complete control of the complex amplitude of electromagnetic field in the region of interest based on high efficiency phase-only metasurfaces [75]. And the polarization can also be manipulated freely by tailoring two orthogonal components of fields simultaneously based on subpixel design strategy in order to obtain holographic images with spatially varied polarization states in microwave frequency. Besides, Song et al. [76] demonstrated a full-polarization reconstructed metasurface that can produce arbitrary polarization for wavefront shaping under linear polarized light illuminating in broadband by utilizing geometric phase principle. The proposed method relies on pixelated metasurfaces, in which each pixel acts as a deflector able to encode both the polarization and the holographic phase information for reconstructing holographic images with arbitrary polarization at specific angle.

Note the polarizations of the above mentioned vetctorial holograms are limited by specific kinds of polarizations. For further increasing the complexity of the polarization pattern, Arbabi et al. [74] demonstrated a vectorial hologram with almost arbitrary polarization patterns using structurally birefringent dielectric metasurfaces by analysing the Stokes parameters. The polarization of light can be fully characterized by using Stokes parameters expressed as follows [21, 22]:

$$
\left\{\begin{array}{c}
S_{0}=I_{0,0}+I_{90,0} \\
S_{1}=S_{0} \cos 2 \chi \cos 2 \psi=I_{0,0}-I_{90,0} \\
S_{2}=S_{0} \cos 2 \chi \sin 2 \psi=I_{45,0}-I_{135,0} \\
S_{3}=S_{0} \sin 2 \chi=I_{45,90}-I_{135,90}
\end{array}\right.
$$


where $X$ indicates the ellipticity angle $(-\pi / 4 \leq X \leq \pi / 4)$ and $\psi(0 \leq \psi \leq \pi)$ represents the orientation angle of polarization ellipse. The $I_{i, j}$ denotes the measured intensities of the transmitted light after passing through the combination of a polarizer and a quarter-wave plate. By using a modified GS algorithm and converting the red-green-blue data of the object to Stokes parameters, the demonstrated metasurfaces present the capable of storing and projecting color image data in the polarization state of a monochromatic hologram as shown in Fig. 3d. Furthermore, Ren et al. [77] demonstrated a 3D vectorial holography where an arbitrary $3 \mathrm{D}$ vectorial field distribution can be precisely reconstructed using the machine learning inverse design with ultrawide viewing angle and high diffraction efficiency by utilizing the designed phase pattern fabricated through 3D direct laser writing.

\section{Angle-multiplexed holography}

Metasurfaces for achieving applications such as holography or beam shaping generally have fixed responses by illuminating at different oblique incident angles, with possible distortions and reductions in efficiency when illumination angles deviate from the design value. However, some efforts have been made to alter this limitation, and make the incident angle become a design freedom for holography or printing-image multiplexing [78-80]. Kamali et al. [78] demonstrated an angle-multiplexed metasurface composed of dielectric U-shaped resonators, which can exhibit independent phase modulations under illumination from two different angles. Because the electric and magnetic resonances within such complex meta-atoms are sensitive to the incident angles, resulting in the large phase differences which can ensure the encoding of independent holograms. As shown in Fig. 4a, the proposed angle-multiplexed hologram can encode and project different holographic images under normal and $30^{\circ}$ illumination angles with TE polarization. Furthermore, a new pixel-design method called the coherent pixel was proposed by Bao et al. [79] for multiple printing-image switching and multiplexing. As shown in Fig. $4 \mathrm{~b}$, the pixel is composed of $n$ elements on sapphire $\left(\mathrm{Al}_{2} \mathrm{O}_{3}\right)$ substrate with same sizes but different position $x_{k}$ and orientation angle $\phi_{k}$. Meanwhile, the intensity of each pixel can be considered as the coherent superposition of contributions by all elements within it expressed by Eq. (4). The coefficient of scattered light of $k t h$ element is represented by $A_{k}$ and the optical parameters incident angle $\theta$, polarization $\sigma$, and wavelength $\lambda$ can be used for the switching process.

$$
I(\theta, \sigma, \lambda)=\left|\sum_{k=1}^{n} A_{k}\left(\sigma, \phi_{k}\right) \exp \left(i 2 \pi \sin \theta x_{k} / \lambda\right)\right|^{2}
$$

Therefore, the independent pixel intensities can be realized under any different arbitrary optical conditions by carefully adjusting the position $x_{k}$ and orientation angle $\phi_{k}$ of each element within one pixel. For the printing-images, their intensity distributions are formed directly on the structure plane and have one-to-one correspondence to the metasurfaces structure. Multiple different images can be switched by simultaneously tailoring the optical parameters including wavelength, incident angle, and polarization of incident light with arbitrary values based on proposed design strategy. Furthermore, Wang et al. [80] demonstrated a dielectric metasurface was capable of realizing simultaneous and independent control of the amplitude, phase, and polarization of 
(a)

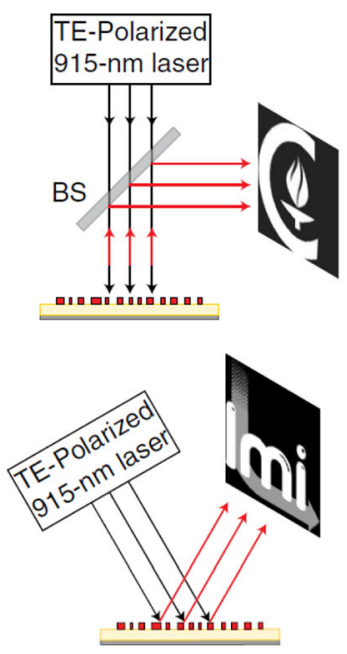

(b)

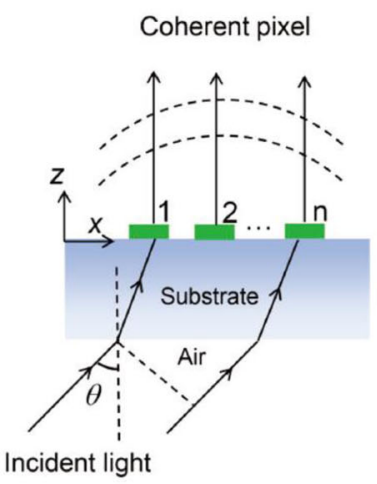

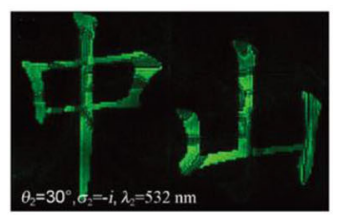

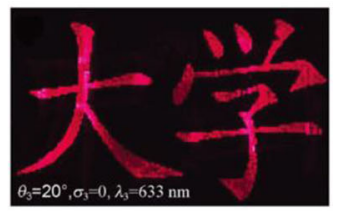

(c)

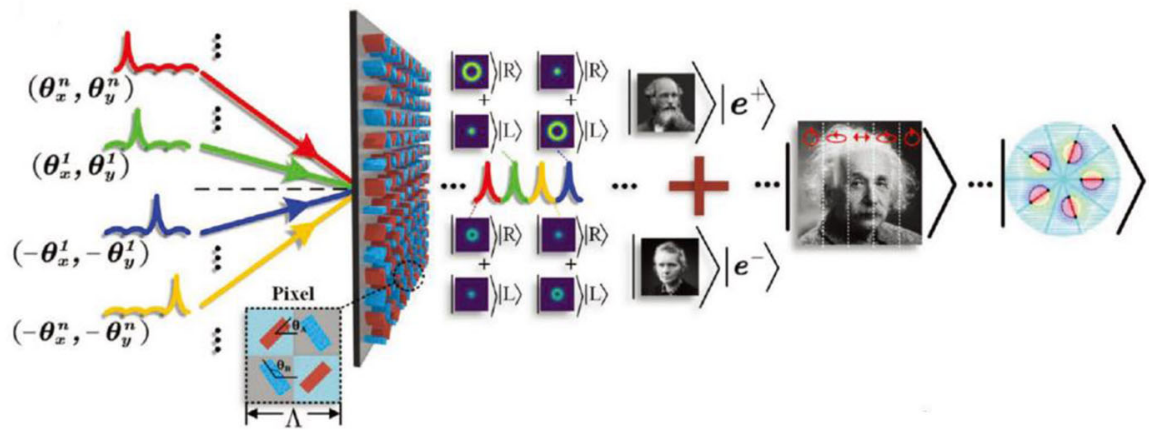

Fig. 4 Angle-multiplexed metasurface holography. a The concept of proposed angle-multiplexed metasurface. Two independent holographic images can be reconstructed under illuminating with TEpolarized light at different incident angle [78]. b Metasurfaces for multiple printing-images switching and encoding based on coherent pixel method. Schematic of the designed coherent pixel is shown on the left panel. The intensity of each pixel can be considered as the coherent superposition of contributions by all elements within it. Three different images can be switched under arbitrary optical conditions based on demonstrated metasurface composed of coherent pixels [79]. c Schematic diagram of the anglemultiplexed metasurface. Different holographic images can be encoded into different polarization channels at target incident angles [80]

output light by combining the coherent pixel method together with geometric phase principle, as shown in Fig. 4c. The proposed method provides a facile route to obtain multichannel, angle-multiplexed and arbitrary spatially varying polarization fields and can be utilized to achieve novel applications such as full Poincaré beams generation, dual-way switching print images, vectorial printing-images, and optical polarization knot profiles generation.

\section{OAM-multiplexed holography}

Vortex beams that carrying orbital angular momentum (OAM) are characterized by a doughnut shaped intensity distribution and exhibit a helical wavefront described by the phase factor $\exp (i l \phi)$, where the unbounded integer $l$ indicates the topological charge number and $\phi$ is the azimuthal angle [81, 82]. As one of the fundamental physical properties of 
light, the OAM mode can be considered as a design freedom for multiplexing strategy due to the infinite topological charge number and orthogonality between different OAM modes. Due to the lack of orbital angular momentum selectivity in the design of conventional holograms, OAM-multiplexing schemes hadn't been achieved in traditional holography before. Recently, Ren et al. [83] solved this problem by spatially sampling the holographic image with an OAM-dependent two-dimensional (2D) Dirac comb function and demonstrated a metasurface OAM holography based on OAM selectivity offered by meta-holograms consisting of GaN nanopillars. As shown in Fig. 5a, three types of meta-holograms with discrete spatial frequency distributions are proposed including OAM-conserving, -selective, and -multiplexing metaholograms. For the OAM-multiplexing metaholograms, OAM beams with different topological charges can reconstruct different images, which may pave the way to achieve ultrahigh-capacity holographic multiplexing and OAM encryption based on metasurfaces. Then Fang et al. [84] successfully extended the OAM-multiplexing metaholograms to be applied in high-security encryption. The design approach is illustrated in Fig. 5b, a 10 bit OAM-multiplexing hologram encoded ten digits of Arabic numerals 0 to 9 is realized when illuminating ten high order OAM modes with topological charge numbers from - 50 to 50, respectively. Two digits of the Arabic numerals from 01 to 26 are used to represent alphabet letters from $\mathrm{A}$ to $\mathrm{Z}$ in order to achieve encrypting messages. Meanwhile, Jin et al. [85] demonstrated a OAM meta-transformer with the capability of transforming the intrinsic phases of OAM $(\exp (i l \hbar))$ of the incidence light into various distinct patterns in the same plane based on carefully designed phase retrieve method, as shown in Fig. 5c. Combining different polarization channels and OAM modes, Zhou et al. [86] proposed a OAMmultiplexing hologram which can reconstruct the OAM selective holographic information only with the exact topological charge and a specific polarization state by using a birefringent metasurface as shown in Fig. 5d. Interestingly, a vortex array with spatially variant topological charge distributions in the cross polarization channel can function as an indicator for the incident beam based on the angular momentum conservation law. Furthermore, a holographic camouflage case for the reconstructed image, in analogy to the well-known stimulated emission depletion (STED) technique in microscopy is realized by using an incident beam with different topological charges as erasers.

\section{Spectral- and spatial-multiplexed metasurface}

Many methods have been proposed to modulate spectral and spatial responses at subwavelength scale simultaneously for achieving color printing and holography within a single metasurfaces in order to enhance the information capacity and security in these years [87-90]. An optical security device with the capability of modulating the phase and amplitude of light was proposed based on integrating a color filter and a phase plate into one pixel for hiding a hologram in the color printing [87]. As shown in Fig. 6a, the device appears as a color image under white light, but reveals three different hidden holographic projections when red, green, and blue laser illumination. Such integrated dual functionalities can provide enhanced security in anti-counterfeiting applications. However, such device utilized direct laser writing method to fabricate arrays of phase plates and color filters with different heights which make the fabrication process become challenging. And the small FOV, undesired multiple diffraction orders and large crosstalk are also need to be improved. For solving above defects, Wei et al. [88] proposed and experimentally demonstrated a meta- 


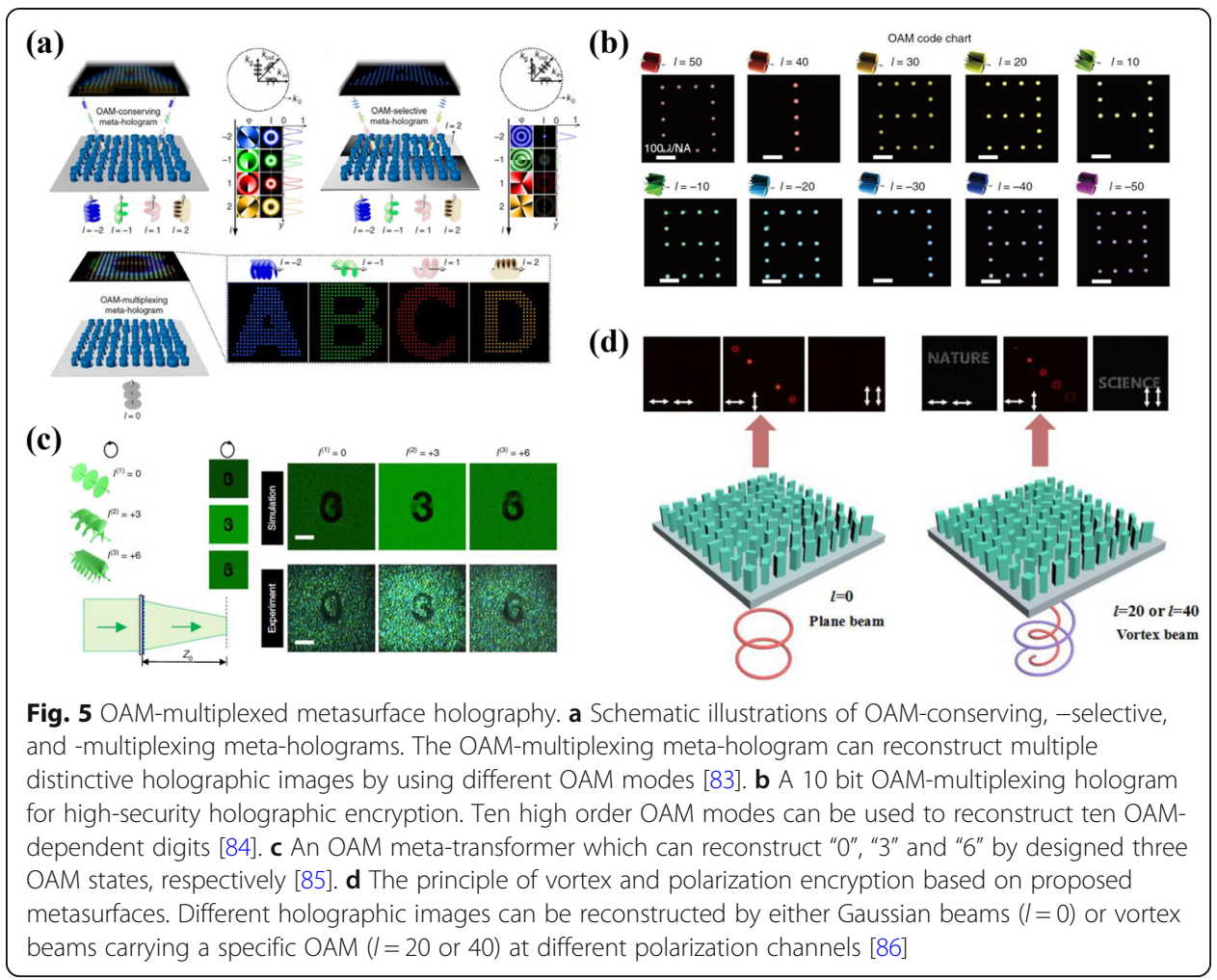

device that integrates color printing and computer-generated holograms within a singlelayer dielectric metasurface by modulating spectral and spatial responses at subwavelength scale simultaneously. As shown in Fig. 6b, optimized amorphous silicon dimers and nanofins are chosen as the meta-atoms to achieve structural color in color printing mode and realize desired phase modulation based on geomertric phase in holographic mode, simultaneously. By utilizing a modified parallel GS algorithm, a microscopic color image (an earth map) is encoded in the designed metasurface, while two different holographic images can reconstructed in the far field under different illumination wavelengths with adequate efficiency and low crosstalk. Meanwhile, the color gamut which is a 2D color space comprised of hue and saturation (HS) is a very important index in display and printing industries. Most of the previous color printing works based on metasurfaces concentrated more on broadening the 2D HS plane while neglecting the brightness control of color which can degrade the performance of color printing. Bao et al. [89] reported a dielectric metasurface made of crystal silicon nanoblocks, which achieved not only tailorable coverage of the primary colors red, green and blue (RGB) but also intensity control of the individual colors in order to achieve arbitrary HSB color nanoprinting and a full-color hologram image simultaneously based on the complex amplitude modulation offered by each unit cell through tailoring the orientation angles $\phi_{1}$ and $\phi_{2}$ based on geometric phase, as shown in Fig. 6c. Furthermore, $\mathrm{Hu}$ et al. [90] demonstrated a 3D-integrated metasurface device by stacking a hologram metasurface on a monolithic Fabry-Pérot cavity-based color filter microarray to simultaneously achieve full-color holography, and microprint as shown in Fig. 6d. The whole device was mainly fabricated by two EBL processes. By utilizing greyscale EBL process the color filters with different heights are fabricated and the hologram metasurface is achieved through an overlay EBL process. Compared to previous work on color holography based on 
(a)
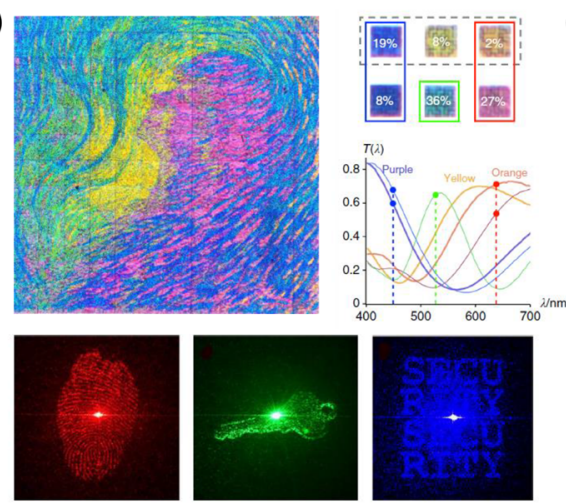

(c)
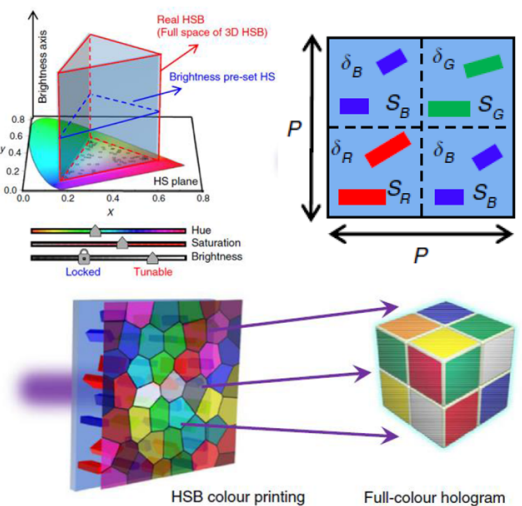

(b)
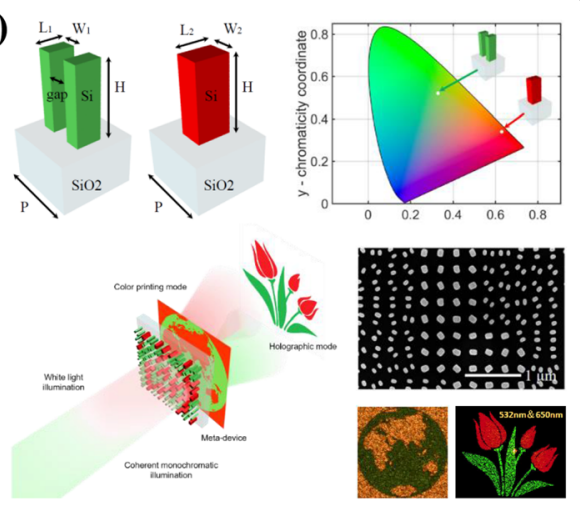

(d)
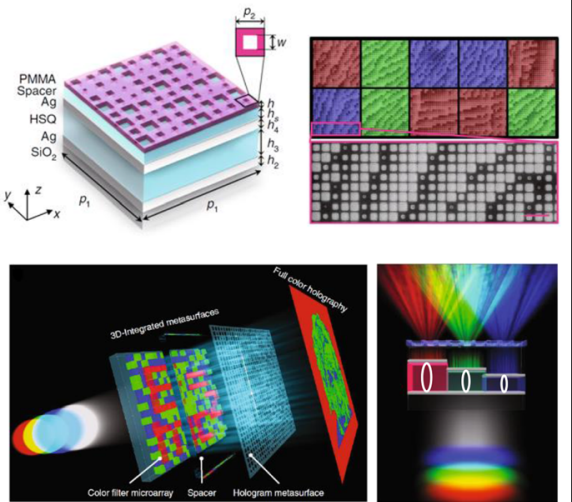

Fig. 6 Spectral- and spatial-multiplexed metasurface. a Enhanced optical security provided by a six-color holographic color print. Luigi Russolo's painting Perfume can be reconstructed under incoherent broadband illumination. Optical micrographs of pillar arrays that produce the colors used in the print are shown on the right panel. Three independent holographic images can be obtained by illuminating with $638 \mathrm{~nm}$ red, $527 \mathrm{~nm}$ green, and $449 \mathrm{~nm}$ blue lasers, respectively [87]. b Schematic illustration of the alldielectric metasurface that integrates dual working modes for incoherent color printing and far-field holography by modulating spatial and spectral responses simultaneously. Selected two types of metaatoms and their calculated structural colors in the CIE 1931 chromaticity diagram are shown on the top panel [88]. c Metasurface for sub-micron resolution HSB color printing and full-color hologram integration. The RGB unit consisting of multiplexed c-silicon nanoblocks for HSB colour tuning is shown on the right top corner [89]. $\mathbf{d}$ Schematic of the 3D-integrated metasurfaces composed of vertically stacking a color-filter microarray with a hologram for achieving full-color holography [90]

metasurfaces, the current integrated device has the advantages of low crosstalk, high efficiency and polarization independence to achieve full-color holography. Meanwhile, the concept of 3D-integrated metasurfaces may lead many multifunctional ultrathin optical systems by stacking more metasurfaces with different functions such as polarizers, wave plates and metalenses.

\section{Other types of metasurface holography}

With the flexibilities supported by the metasurface platform, many efforts have been devoted to achieve metasurface holography with multifunctional or tunable properties for realizing future practical applications. In this section, we will discuss recent developments in tunable holography, nonlinear holography, Janus (or directional related) and bilayer metasurfaces holography. 


\section{Tunable metasurface holography}

Tunable or dynamic control of metasurface holograms are desirable for practical display applications. While, most of the reported metasurface holograms are static or only present several different states by above mentioned multiplexing methods. Recently, some programmable metasurfaces are proposed in GHz region [91-93]. However, the limitation of current fabrication technology make it difficult to adopt the same strategy in the progress of designing dynamic metasurfaces for visible light. While some previous works have still been made to fulfill tunable control of metasurface holograms in visible range, by utilizing the principle of phase change materials (PCMs), stretching the stretchable substrate or chemical reactions [94-96].

GeSbTe (GST) is a kind of PCMs which can be widely used in optical storage and reconfigurable photonic devices [97-99]. By appropriate thermal, optical, or electrical stimulus, GST can be repeatedly switched between amorphous and crystalline states and exhibit different refractive index with high contrast in the near- and middle-infrared spectral range. Combining plasmonic metasurfaces with GST, switchable metasurfaces for the purpose of achieving spin Hall effect, vortex beam generation and holography are demonstrated when the GST is in the amorphous state as shown in Fig. 7a [94]. When heating these devices, the GST changed to crystalline state and these functionalities disappeared. Meanwhile, stretching the stretchable substrate with a hologram on it provides another method to achieve tunable metasurfaces holography. Malek et al. [95] demonstrated a reconfigurable metasurface hologram composed of gold nanorods on a stretchable polydimethylsiloxane (PDMS). When stretching the hologram with a stretch ratio $s$, the electric field after stretching can be expressed by Eq. (5).

$$
E^{\prime}\left(x^{\prime}, y^{\prime}, z^{\prime}\right)=E^{\prime}\left(s x, s y, s^{2} z\right)=E(x, y, z) e^{i k\left(s^{2}-1\right) z}
$$

We can find that the holographic image is also stretched by the factor $s$ while the image plane is moved to the position $s^{2} z$. Based on this principle, different images can be obtained in the same plane by stretching the substrate with different stretch ratio $s$ as shown in Fig. 7b. Furthermore, by applying the hydrodynamic process of magnesium $(\mathrm{Mg})$ through controlled chemical reactions, a kind of addressable metasurfaces have been demonstrated [96]. Mg exhibits excellent plasmonic properties at visible frequencies and can also undergo a chemical reaction from metal to dielectric through hydrogen loading, forming magnesium hydride $\left(\mathrm{MgH}_{2}\right)$. Such reaction can be reversible by dehydrogenation using oxygen. By utilizing this feature, the plasmonic response (scattered intensity) of $\mathrm{Mg}$ nanorod can be reversibly switched between on and off state which leading the dynamic holography as presented in Fig. 7c. The Cr capping layer of $\mathrm{Mg} / \mathrm{Pd} / \mathrm{Cr}$ nanorod can effectively slow down both the hydrogenation and dehydrogenation rates and provide more switchable states which result in achieving optical information processing and encryption.

\section{Nonlinear metasurface holography}

Apart from light modulation in linear optics, metasurfaces have gradually shown their great potential of tailoring the wavefront in nonlinear optical regimes. Numerous methods have been proposed for achieving nonlinear wavefront control such as harmonic generation [18], 
(a)
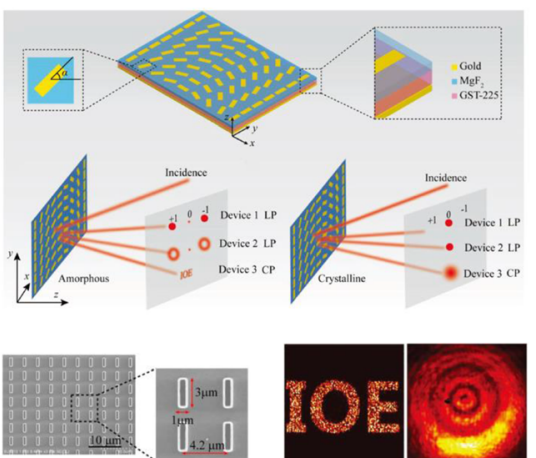

(d)

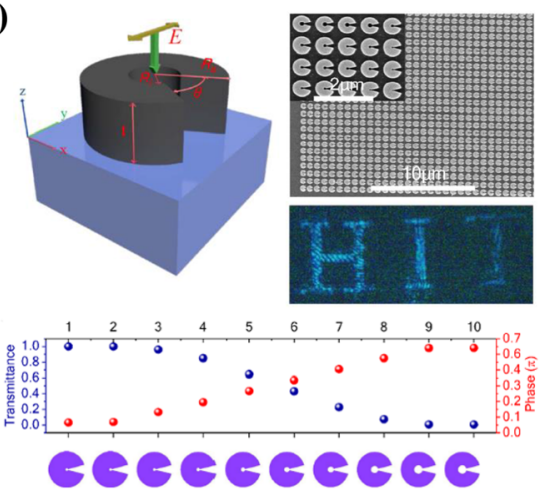

(b)

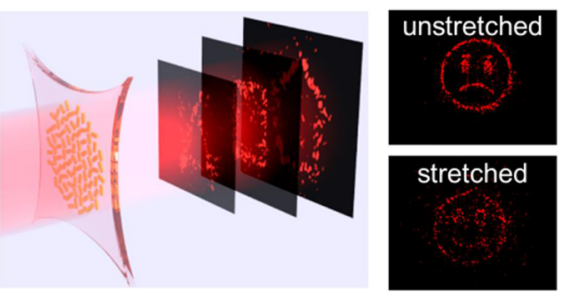

(c)

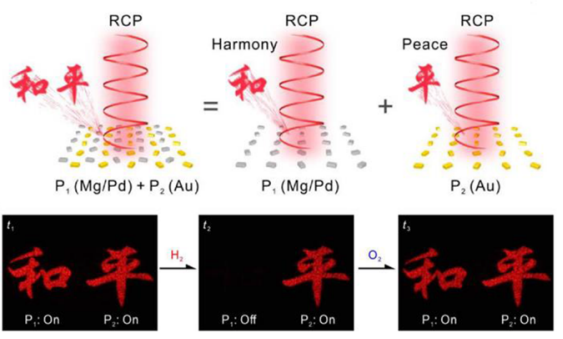

(e)
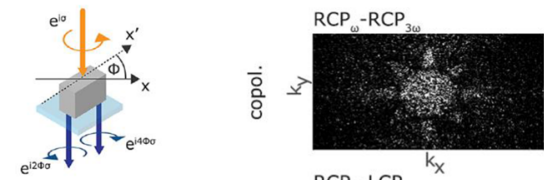

$R C P-L C P$

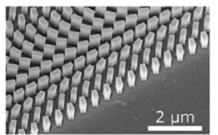

Fig. 7 Tunable metasurface holography and nonlinear metasurface holography. a Schematic of the demonstrated switchable photonic SOls. Different optical performances of three designed metadevices can be switched when the GST layer is in amorphous or crystalline states. SEM images of fabricated patch antennas array are shown on the left bottom corner. The experimental results are shown on the right bottom corner [94]. b Schematic of a metasurface hologram on a stretchable substrate. The sizes and locations of holographic images can be changed by stretching the substrate [95]. c Merged metasurfaces composed of two independent phase profiles containing $\mathrm{Mg} / \mathrm{Pd}$ and Au nanorods as dynamic and static pixels, respectively. The representative snapshots of the holographic images harmony and peace during hydrogenation and dehydrogenation are shown on the bottom [96]. d All-dielectric metasurfaces composed of C-shaped Si nanoantennas for nonlinear holography at THG [100]. e Silicon metasurfaces for nonlinear wavefront control of the third-harmonic generation and multiplexed holography. The generated nonlinear signal acquires phase modulations of $\theta_{c o}=2 \sigma \varphi$ and $\theta_{\text {cross }}=4 \sigma \varphi$ in co- and cross-polarization states controlled by the oreientation angle, respectively. The experimental results of multiplexed holography are shown on the right [101]

nonlinear imaging $[102,103]$, nonlinear beam shaping $[42,104-106]$ and holography $[19$, $100,101,107-109]$ in recent years.

Benefitting from the continuous phase control of the fundamental and harmonic generation waves based on nonlinear PB phase, Ye et al. [19] reported a spin and wavelength multiplexed nonlinear metasurface holography with the capability of encoding different phase profiles into fundamental and harmonic generation waves in different spin channels. However, high dissipative losses and a low optical damage threshold of plasmonic metasurfaces severely restrict the obtainable nonlinear conversion efficiency. Therefore, all-dielectric metasurfaces have gradually become suitable devices for achieving nonlinear wavefront control due to they can overcome above limitations and realize larger nonlinear conversion efficiencies. Gao et al. [100] demonstrated an all-dielectric metasurface which composed of C-shaped Si nanoantennas for achieving nonlinear holography. The magnetic resonance and electric resonance of the nanoantennas at fundamental wavelength can significantly enhance the third-harmonic generation (THG) process, whereas the 
higher order resonances at THG wavelengths can suppress the absorption loss of THG signals. Meanwhile, the phase modulation of THG signal can cover 0 to $2 \pi$ by varying the structural parameters of the C elements in order to realize THG holograms at two different fundamental wavelengths. Furthermore, Reineke et al. [101] provided an approach to encode phase gradients and holographic images on $\mathrm{C} 2$ nanostructured silicon metasurfaces based on geometric phase principle with high damage thresholds as shown in Fig. $7 \mathrm{~d}$. The generated nonlinear signal acquires phase modulations of $\theta_{c o}=2 \sigma \phi$ and $\theta_{\text {cross }}=4 \sigma \phi$ in co- and cross-polarization states controlled by the orientation angle $\phi$ as well as obeying the selection rule, respectively. Different high fidelity holographic images can be obtained by utilizing the polarization states of the THG.

Recently, $\mathrm{Hu}$ et al. [106] demonstrated a synthetic metasurface composed of a plasmonic Au metasurface and the monolayer two-dimensional transition metal dichalcogenides (TMDCs) with the capability of steering the nonlinear photons from different valleys to any desired direction in free space at room temperature based on critical spin-valley locked nonlinear selection rule. An enhancement of the SHG by one order of magnitude is achieved owing to large plasmonic field localization around the nanoholes of Au metasurface. Then the proposed design strategy is extended to realize more complicated nonlinear wavefront functionalities such as second-harmonic (SH) OAM generation, versatile polarization control and holograms [109].

\section{Janus and bilayer metasurfaces holography}

Janus is depicted as the god of beginnings who has two faces looking into the past and future in Roman mythology. For the purpose of achieving metasurface holograms with more fancy functionalities the concepts of asymmetric and bidirectional metasurfaces (called as Janus metasurfaces) are proposed. As well known, single layer transmitted metasurfaces usually exhibit identical electromagnetic (EM) functionalities when changing the propagation direction of incident light based on reciprocal law, which make the directionality features unexploited. For breaking this limitation, some efforts have been devoted in recent years [110-112].

Chen et al. [110] reported a direction-controlled polarization-encrypted data storage based on 3D Janus plasmonic helical nanoapertures which can be fabricated by using one-step grayscale focused ion beam milling method. In forward direction, circular dichroism is achieved in experiment owing to spin-dependent mode coupling process inside helical nanoaperture. When changing to the opposite propagation direction, the circular dichroism disappears but the plasmonic helical nanoapertures exhibit giant linear dichroism. By utilizing these properties, bidirectional imaging is successfully demonstrated based on designed Janus metasurfaces which composed of two enantiomers of helical nanoapertures as shown in Fig. 8a. A binary QR code image can be obtained in forward direction under certain helicity of circularly polarized light. Note here only the intensity pattern from each pixels can be modulated. In contrast, when illuminate linear polarized light from backward direction, a distinct grayscale image is generated based on Malus's law and giant linear dichroism. By analyzing the S-parameters of transmitted light, metasurfaces composed of cascaded subwavelength anisotropic impedance sheets with the capacity of operating for unidirectional forward and backward transmission with desired phase functions are realized in $\mathrm{GHz}$ region and can further 
(a)
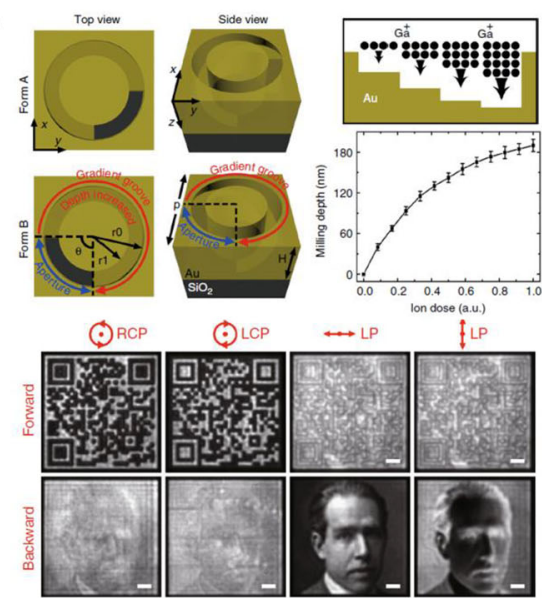

(b)
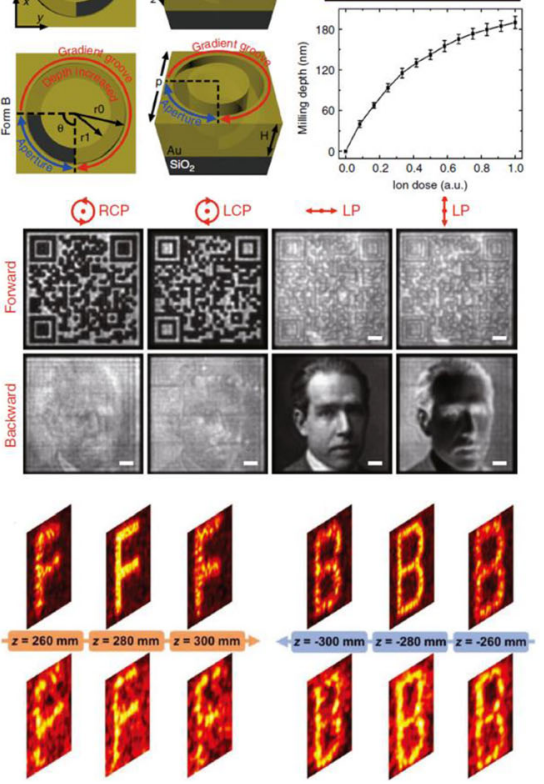

(c)
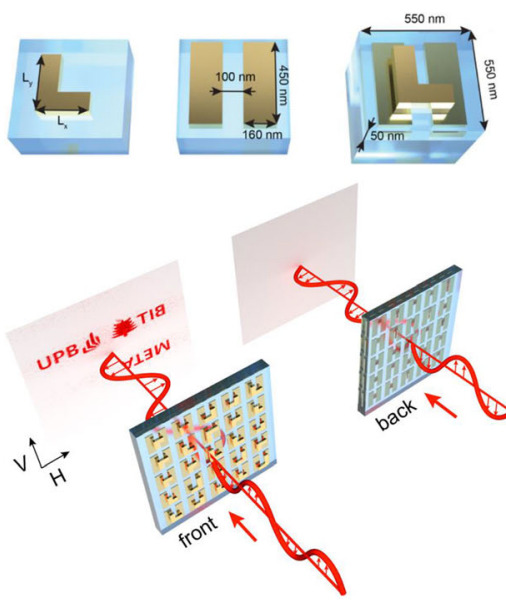

(d)

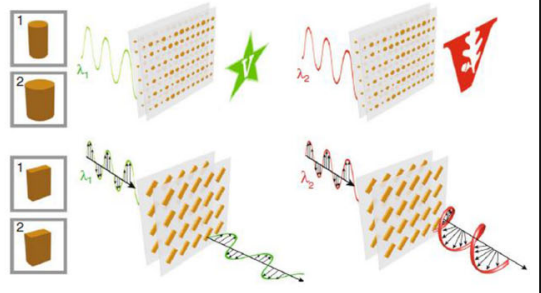

Fig. 8 Janus and bilayer metasurfaces holography. a Direction-controlled polarization-encrypted data storage based on Janus metasurface composed of two enantiomeric plasmonic helical nanoapertures. Experimental results of the Janus metasurface in both the forward and backward directions for various incident polarizations are shown on the bottom [110]. b Simulated and experimental results of proposed directional Janus metasurface observed at different observation planes at $8.6 \mathrm{GHz}$ for forward and backward waves [111]. c Schematic illustration of cascaded two-layer polarization sensitive meta-hologram. The unitcell composed of $L$ shape antenna and dimer layer are shown on the top [112]. $\mathbf{d}$ Schematic of multiwavelength holograms, multi-wavelength waveplates, and 3D holograms based on dielectric bilayer metasurfaces [113]

be used to design Janus metasurfaces by multiplexing [111]. The proposed Janus metasurfaces can break out-of-plane symmetry and enable different functions for opposite propagation directions and realized functionalities including one-way anomalous refraction, one-way focusing, asymmetric focusing, and direction-controlled holograms as shown in Fig. 8b.

Furthermore, cascaded metasurfaces with direction related functionalities in NIR range have emerged recently. Frese et al. [112] presented a two-layer plasmonic metasurface which can break the spatial symmetry for propagation and generate a bidirectional functionality with full phase and amplitude control of the transmitted light. Such metasurface is composed of cascaded L-shape antenna and dimer layer, where the L-shape mainly contribute to the polarization conversion and phase modulation, while the dimer can result in the selection of polarization. Schematic illustration of such asymmetry transmission functionality of the proposed polarization sensitive meta-hologram is shown in Fig. 8c. The encoded hologram is designed to appear in a particular linear cross-polarization channel, while it disappears in the reverse propagation direction. Apart from plasmonic counterparts, Zhou et al. [113] explored cascaded dielectric metasurfaces which expand the design space for meta-optics. The coupling between the two layers can be designed to minimize, and the ability to independently control the geometry and function of each layer enables the development of multifunctional metaoptics in which two or more optical 
properties are independently designed. As a proof of concept, multiwavelength holograms, multiwavelength waveplates, and polarization-insensitive 3D holograms were demostrated based on phase and amplitude masks shown in Fig. 8d.

\section{Conclusions and outlook}

In summary, we have reviewed recent development of metasurface holography. The metasurfaces can provide massive design freedoms for modulating the phase, amplitude, polarization together with multiple parameters of light within subwavlength resolution. Such appealing features make metasurface holography exhibit superior performance over conventional holography based on SLM or diffractive optical elements. Holographic images with high quality and high resolution can be obtained at large FOV. Meanwhile, machine learning and inverse design methods are penetrated in the field and can help researchers design structures with specified optical responses or metasurfaces with desired functionalities. Some pioneering works have been reported [114-117], which may provide powerful design strategies for multiple functionalities. Nevertheless, several challenges are still remain in the terms of multi-dimensional manipulation, dynamic holographic display and large area fabrication.

The dynamic control of the holograms is essentially required for achieving ultimate holographic display. While, the functionalities of most current metasurfaces holograms are static after fabrication. Although new techniques for dynamic control are proposed based on reprogrammable coding metasurfaces, phase change materials or chemical reaction, it is still very difficult to acquire arbitrarily tunable wavefront modulation in real time and achieve dynamic display in visible region. With the rapid development of nanofabrication technologies and gradually mature metasurfaces design strategies, we hope this problem will be solved in the near future. Furthermore, by utilizing the DMD to generate time sequence beams to selectively excite the corresponding sub meta holograms, dynamic display with $2^{28}$ different holographic images and an extremely high frame rate (9523 frames per second) in the visible range have been demonstrated [118]. The proposed method provided an alternative method to achieve dynamic display.

Some practical compact devices such as spectrometer [119], full-Stokes polarization camera [120], quantitative phase gradient microscope (QGPM) [121] and cold atoms generation [122] are deomonstrated based on single or several cascaded metasurfaces in recent years. The design strategies of metasurface holography can also be extended to achieve optical elements with specific functionalities such as beam shaping, beam splitting, parallel processing for laser fabrication or optical tweezers, and even all optical diffractive deep neural networks, which may provide a powerful tool for realizing compact optical systems with smart abilities.

Acknowledgements

Not applicable.

Authors' contributions

RZ prepared the manuscript. LH and YW revised the manuscript. The author(s) read and approved the final manuscript.

Funding

National Key Research and Development Program of China 2017YFB1002900, Ministry of Science and Technology,

China; Fok Ying-Tong Education Foundation of China (161009); Beijing Nova Program (Z171100001117047); Natural Science Foundation of Beijing Municipality (4172057); National Natural Science Foundation of China (61775019); Beijing Outstanding Young Scientist Program (BJJWZYJH01201910007022). 


\section{Availability of data and materials}

The datasets and figures used and analyzed during the current study are available from the corresponding author on reasonable request.

\section{Competing interests}

The authors declare that they have no competing interests.

\section{Received: 21 August 2020 Accepted: 30 September 2020}

Published online: 19 October 2020

\section{References}

1. Gabor D. A new microscopic principle. Nature. 1948;161:777-8.

2. Brown BR, Lohmann AW. Complex spatial filtering with binary masks. Appl Opt. 1966;5:967-9.

3. Gabor D. Holography, 1948-1971. Proc IEEE. 1972;60:655-68.

4. Fukushima S, Kurokawa T, Ohno M. Real-time hologram construction and reconstruction using a high-resolution spatial light-modulator. Appl Phys Lett. 1991;58:787-9.

5. Jiang Q, Jin G, Cao L. When metasurface meets hologram: principle and advances. Adv Opt Photon. 2019;11:518.

6. Sung J, Lee G-Y, Lee B. Progresses in the practical metasurface for holography and lens. Nanophotonics. 2019;8:1701-18.

7. Huang L, Zhang S, Zentgraf T. Metasurface holography: from fundamentals to applications. Nanophotonics. 2018;7: 1169-90.

8. Lee GY, Sung J, Lee B. Recent advances in metasurface hologram technologies (invited paper). ETRI J. 2019;41:10-22.

9. Genevet P, Capasso F. Holographic optical metasurfaces: a review of current progress. Rep Prog Phys. 2015;78:024401.

10. Xu Z, Huang L, Li X, Tang C, Wei Q, Wang Y. Quantitatively correlated amplitude holography based on photon sieves. Adv Opt Mater. 2019:8:1901169.

11. Huang K, Liu H, Garcia-Vidal FJ, Hong M, Luk'yanchuk B, Teng J, Qiu C-W. Ultrahigh-capacity non-periodic photon sieves operating in visible light. Nat Commun. 2015;6:7059.

12. Li J, Zhang Y, Li J, Yan X, Liang L, Zhang Z, Huang J, Li J, Yang Y, Yao J. Amplitude modulation of anomalously reflected terahertz beams using all-optical active Pancharatnam-berry coding metasurfaces. Nanoscale. 2019;11:5746-53.

13. Yu N, Genevet P, Kats MA, Aieta F, Tetienne J-P, Capasso F, Gaburro Z. Light propagation with phase discontinuities: generalized laws of reflection and refraction. Science. 2011;334:333-7.

14. Sun S, He Q, Xiao S, Xu Q, Li X, Zhou L. Gradient-index meta-surfaces as a bridge linking propagating waves and surface waves. Nat Mater. 2012;11:426-31.

15. Huang L, Chen X, Mühlenbernd H, Li G, Bai B, Tan Q, Jin G, Zentgraf T, Zhang S. Dispersionless phase discontinuities for controlling light propagation. Nano Lett. 2012;12:5750-5.

16. Kang M, Feng T, Wang H-T, Li J. Wave front engineering from an array of thin aperture antennas. Opt Express. 2012;20: 15882-90.

17. Decker M, Staude I, Falkner M, Dominguez J, Neshev DN, Brener I, Pertsch T, Kivshar YS. High-efficiency dielectric Huygens' surfaces. Adv Opt Mater. 2015;3:813-20.

18. Li G, Chen S, Pholchai N, Reineke B, Wong PWH, Pun EYB, Cheah KW, Zentgraf T, Zhang S. Continuous control of the nonlinearity phase for harmonic generations. Nat Mater. 2015;14:607-12.

19. Ye W, Zeuner F, Li X, Reineke B, He S, Qiu C-W, Liu J, Wang Y, Zhang S, Zentgraf T. Spin and wavelength multiplexed nonlinear metasurface holography. Nat Commun. 2016;7:11930.

20. Aieta F, Genevet P, Kats MA, Yu N, Blanchard R, Gaburro Z, Capasso F. Aberration-free ultrathin flat lenses and axicons at telecom wavelengths based on plasmonic metasurfaces. Nano Lett. 2012;12:4932-6.

21. Kruk S, Hopkins B, Kravchenko II, Miroshnichenko A, Neshev DN, Kivshar YS. Invited article: broadband highly efficient dielectric metadevices for polarization control. APL Photonics. 2016;1:030801.

22. Li T, Huang L, Liu J, Wang Y, Zentgraf T. Tunable wave plate based on active plasmonic metasurfaces. Opt Express. 2017; 25:4216-26.

23. Shalaev MI, Sun J, Tsukernik A, Pandey A, Nikolskiy K, Litchinitser NM. High-efficiency all-dielectric metasurfaces for ultracompact beam manipulation in transmission mode. Nano Lett. 2015;15:6261-6.

24. Devlin RC, Ambrosio A, Rubin NA, Mueller JB, Capasso F. Arbitrary spin-to-orbital angular momentum conversion of light. Science. 2017;358:896-901.

25. Sroor H, Huang Y-W, Sephton B, Naidoo D, Vallés A, Ginis V, Qiu C-W, Ambrosio A, Capasso F, Forbes A. High-purity orbital angular momentum states from a visible metasurface laser. Nat Photonics. 2020;14:498-503.

26. Arbabi A, Horie Y, Bagheri M, Faraon A. Dielectric metasurfaces for complete control of phase and polarization with subwavelength spatial resolution and high transmission. Nat Nanotechnol. 2015;10:937-43.

27. Mueller JB, Rubin NA, Devlin RC, Groever B, Capasso F. Metasurface polarization optics: independent phase control of arbitrary orthogonal states of polarization. Phys Rev Lett. 2017;118:113901.

28. Deng Z-L, Deng J, Zhuang X, Wang S, Li K, Wang Y, Chi Y, Ye X, Xu J, Wang GP. Diatomic metasurface for vectorial holography. Nano Lett. 2018;18:2885-92.

29. Deng ZL, Jin M, Ye X, Wang S, Shi T, Deng J, Mao N, Cao Y, Guan BO, Alù A. Full-color complex-amplitude vectorial holograms based on multi-freedom metasurfaces. Adv Funct Mater. 2020:30:1910610.

30. Bao Y, Ni J, Qiu CW. A minimalist single-layer metasurface for arbitrary and full control of vector vortex beams. Adv Mater. 2020;32:1905659.

31. Chen WT, Khorasaninejad M, Zhu AY, Oh J, Devlin RC, Zaidi A, Capasso F. Generation of wavelength-independent subwavelength Bessel beams using metasurfaces. Light Sci Appl. 2017;6:e16259-e59.

32. Yue F, Wen D, Xin J, Gerardot BD, Li J, Chen X. Vector vortex beam generation with a single plasmonic metasurface. ACS Photonics. 2016;3:1558-63.

33. Song X, Huang L, Sun L, Zhang X, Zhao R, Li X, Wang J, Bai B, Wang Y. Near-field plasmonic beam engineering with complex amplitude modulation based on metasurface. Appl Phys Lett. 2018;112:073104. 
34. Lin Z, Li X, Zhao R, Song X, Wang Y, Huang L. High-efficiency Bessel beam array generation by Huygens metasurfaces. Nanophotonics. 2019:8:1079-85.

35. Chen X, Huang L, Mühlenbernd H, Li G, Bai B, Tan Q, Jin G, Qiu C-W, Zhang S, Zentgraf T. Dual-polarity plasmonic metalens for visible light. Nat Commun. 2012;3:1198.

36. Khorasaninejad M, Chen WT, Devlin RC, Oh J, Zhu AY, Capasso F. Metalenses at visible wavelengths: diffraction-limited focusing and subwavelength resolution imaging. Science. 2016;352:1190-4.

37. Chen WT, Zhu AY, Sanjeev V, Khorasaninejad M, Shi Z, Lee E, Capasso F. A broadband achromatic metalens for focusing and imaging in the visible. Nat Nanotechnol. 2018;13:220-6.

38. Wang S, Wu PC, Su V-C, Lai Y-C, Chen M-K, Kuo HY, Chen BH, Chen YH, Huang T-T, Wang J-H. A broadband achromatic metalens in the visible. Nat Nanotechnol. 2018:13:227-32.

39. Shrestha S, Overvig AC, Lu M, Stein A, Yu N. Broadband achromatic dielectric metalenses. Light Sci Appl. 2018;7:1-11.

40. Wang Z, Jia H, Yao K, Cai W, Chen H, Liu Y. Circular dichroism metamirrors with near-perfect extinction. ACS Photonics. 2016:3:2096-101.

41. Zhu AY, Chen WT, Zaidi A, Huang Y-W, Khorasaninejad M, Sanjeev V, Qiu C-W, Capasso F. Giant intrinsic chiro-optical activity in planar dielectric nanostructures. Light Sci Appl. 2018;7:17158.

42. Camacho-Morales R, Rahmani M, Kruk S, Wang L, Xu L, Smirnova DA, Solntsev AS, Miroshnichenko A, Tan HH, Karouta F. Nonlinear generation of vector beams from AlGaAs nanoantennas. Nano Lett. 2016;16:7191-7.

43. Yang Y, Wang W, Boulesbaa A, Kravchenko II, Briggs DP, Puretzky A, Geohegan D, Valentine J. Nonlinear Fano-resonant dielectric metasurfaces. Nano Lett. 2015;15:7388-93.

44. Franklin D, Chen Y, Vazquez-Guardado A, Modak S, Boroumand J, Xu D, Wu S-T, Chanda D. Polarization-independent actively tunable colour generation on imprinted plasmonic surfaces. Nat Commun. 2015;6:7337.

45. Duan X, Kamin S, Liu N. Dynamic plasmonic colour display. Nat Commun. 2017:8:14606.

46. Huang L, Chen X, Mühlenbernd H, Zhang H, Chen S, Bai B, Tan Q, Jin G, Cheah K-W, Qiu C-W. Three-dimensional optical holography using a plasmonic metasurface. Nat Commun. 2013:4:2808.

47. Zheng G, Mühlenbernd H, Kenney M, Li G, Zentgraf T, Zhang S. Metasurface holograms reaching 80\% efficiency. Nat Nanotechnol. 2015;10:308-12.

48. Huang L, Mühlenbernd H, Li X, Song X, Bai B, Wang Y, Zentgraf T. Broadband hybrid holographic multiplexing with geometric metasurfaces. Adv Mater. 2015;27:6444-9.

49. Wei Q, Huang L, Li X, Liu J, Wang Y. Broadband multiplane holography based on plasmonic metasurface. Adv Opt Mater. 2017:5:1700434.

50. Kim I, Yoon G, Jang J, Genevet P, Nam KT, Rho J. Outfitting next generation displays with optical metasurfaces. ACS Photonics. 2018;5:3876-95.

51. Yu N, Capasso F. Flat optics with designer metasurfaces. Nat Mater. 2014:13:139-50.

52. Kildishev AV, Boltasseva A, Shalaev VM. Planar photonics with metasurfaces. Science. 2013;339:1232009.

53. Lin D, Fan P, Hasman E, Brongersma ML. Dielectric gradient metasurface optical elements. Science. 2014;345:298-302.

54. Wang L, Kruk S, Tang H, Li T, Kravchenko I, Neshev DN, Kivshar YS. Grayscale transparent metasurface holograms. Optica. 2016:3:1504-5.

55. Devlin RC, Khorasaninejad M, Chen WT, Oh J, Capasso F. Broadband high-efficiency dielectric metasurfaces for the visible spectrum. Proc Natl Acad Sci. 2016;113:10473-8.

56. Jahani S, Jacob Z. All-dielectric metamaterials. Nat Nanotechnol. 2016;11:23-36.

57. Butt H, Montelongo Y, Butler T, Rajesekharan R, Dai Q, Shiva-Reddy SG, Wilkinson TD, Amaratunga GA. Carbon nanotube based high resolution holograms. Adv Mater. 2012;24:OP331-OP36.

58. Walther B, Helgert C, Rockstuhl C, Setzpfandt F, Eilenberger F, Kley EB, Lederer F, Tünnermann A, Pertsch T. Spatial and spectral light shaping with metamaterials. Adv Mater. 2012;24:6300-4.

59. Lee G-Y, Yoon G, Lee S-Y, Yun H, Cho J, Lee K, Kim H, Rho J, Lee B. Complete amplitude and phase control of light using broadband holographic metasurfaces. Nanoscale. 2018;10:4237-45.

60. Overvig AC, Shrestha S, Malek SC, Lu M, Stein A, Zheng C, Yu N. Dielectric metasurfaces for complete and independent control of the optical amplitude and phase. Light Sci Appl. 2019:8:92.

61. Ni X, Kildishev AV, Shalaev VM. Metasurface holograms for visible light. Nat Commun. 2013;4:2807.

62. Wang Q, Zhang X, Xu Y, Gu J, Li Y, Tian Z, Singh R, Zhang S, Han J, Zhang W. Broadband metasurface holograms: toward complete phase and amplitude engineering. Sci Rep. 2016;6:32867.

63. Huang Y-W, Chen WT, Tsai W-Y, Wu PC, Wang C-M, Sun G, Tsai DP. Aluminum plasmonic multicolor meta-hologram. Nano Lett. 2015;15:3122-7.

64. Wang B, Dong F, Li Q-T, Yang D, Sun C, Chen J, Song Z, Xu L, Chu W, Xiao Y-F. Visible-frequency dielectric metasurfaces for multiwavelength achromatic and highly dispersive holograms. Nano Lett. 2016;16:5235-40.

65. Jin L, Dong Z, Mei S, Yu YF, Wei Z, Pan Z, Rezaei SD, Li X, Kuznetsov Al, Kivshar YS. Noninterleaved metasurface for $\left(2^{6}-1\right)$ spin-and wavelength-encoded holograms. Nano Lett. 2018;18:8016-24.

66. Li X, Chen L, Li Y, Zhang X, Pu M, Zhao Z, Ma X, Wang Y, Hong M, Luo X. Multicolor 3D meta-holography by broadband plasmonic modulation. Sci Adv. 2016;2:e1601102.

67. Wang B, Dong F, Yang D, Song Z, Xu L, Chu W, Gong Q, Li Y. Polarization-controlled color-tunable holograms with dielectric metasurfaces. Optica. 2017;4:1368-71.

68. Hu Y, Li L, Wang Y, Meng M, Jin L, Luo X, Chen Y, Li X, Xiao S, Wang H. Trichromatic and tripolarization-channel holography with noninterleaved dielectric metasurface. Nano Lett. 2019;20:994-1002.

69. Hu Y, Wang $X$, Luo $X$, Ou X, Li L, Chen Y, Yang P, Wang S, Duan H. All-dielectric metasurfaces for polarization manipulation: principles and emerging applications. Nanophotonics. 2020:20200220.

70. Intaravanne $Y$, Chen $X$. Recent advances in optical metasurfaces for polarization detection and engineered polarization profiles. Nanophotonics. 2020;9:1003.

71. Montelongo Y, Tenorio-Pearl JO, Milne WI, Wilkinson TD. Polarization switchable diffraction based on subwavelength plasmonic nanoantennas. Nano Lett. 2014;14:294-8.

72. Wen D, Yue F, Li G, Zheng G, Chan K, Chen S, Chen M, Li KF, Wong PWH, Cheah KW. Helicity multiplexed broadband metasurface holograms. Nat Commun. 2015;6:8241. 
73. Zhao R, Sain B, Wei Q, Tang C, Li X, Weiss T, Huang L, Wang Y, Zentgraf T. Multichannel vectorial holographic display and encryption. Light Sci Appl. 2018;7:95.

74. Arbabi E, Kamali SM, Arbabi A, Faraon A. Vectorial holograms with a dielectric metasurface: ultimate polarization pattern generation. ACS Photonics. 2019;6:2712-8.

75. Wu J, Wang Z, Fang Z, Liang J, Fu X, Liu J, Wu H, Bao D, Miao L, Zhou X, Cheng Q, Cui T. Full-state synthesis of electromagnetic fields using high efficiency phase-only metasurfaces. Adv Funct Mater. 2020:2004144.

76. Song Q, Baroni A, Sawant R, Ni P, Brandli V, Chenot S, Vézian S, Damilano B, de Mierry P, Khadir S. Ptychography retrieval of fully polarized holograms from geometric-phase metasurfaces. Nat Commun. 2020;11:2651.

77. Ren H, Shao W, Li Y, Salim F, Gu M. Three-dimensional vectorial holography based on machine learning inverse design. Sci Adv. 2020;6:eaaz4261.

78. Kamali SM, Arbabi E, Arbabi A, Horie Y, Faraji-Dana M, Faraon A. Angle-multiplexed metasurfaces: encoding independent wavefronts in a single metasurface under different illumination angles. Phys Rev X. 2017;7:041056.

79. Bao Y, Yu Y, Xu H, Lin Q, Wang Y, Li J, Zhou ZK, Wang XH. Coherent pixel design of metasurfaces for multidimensional optical control of multiple printing-image switching and encoding. Adv Funct Mater. 2018;28:1805306.

80. Wang E, Niu J, Liang Y, Li H, Hua Y, Shi L, Xie C. Complete control of multichannel, angle-multiplexed, and arbitrary spatially varying polarization fields. Adv Opt Mater. 2020;8:1901674.

81. Wang J, Yang J-Y, Fazal IM, Ahmed N, Yan Y, Huang H, Ren Y, Yue Y, Dolinar S, Tur M. Terabit free-space data transmission employing orbital angular momentum multiplexing. Nat Photonics. 2012;6:488-96.

82. Shen Y, Wang X, Xie Z, Min C, Fu X, Liu Q, Gong M, Yuan X. Optical vortices 30 years on: OAM manipulation from topological charge to multiple singularities. Light Sci Appl. 2019;8:90.

83. Ren H, Briere G, Fang X, Ni P, Sawant R, Héron S, Chenot S, Vézian S, Damilano B, Brändli V. Metasurface orbital angular momentum holography. Nat Commun. 2019;10:2986.

84. Fang X, Ren H, Gu M. Orbital angular momentum holography for high-security encryption. Nat Photonics. 2020; $14: 102-8$.

85. Jin L, Huang Y-W, Jin Z, Devlin RC, Dong Z, Mei S, Jiang M, Chen WT, Wei Z, Liu H. Dielectric multi-momentum metatransformer in the visible. Nat Commun. 2019;10:4789.

86. Zhou H, Sain B, Wang Y, Schlickriede C, Zhao R, Zhang X, Wei Q, Li X, Huang L, Zentgraf T. Polarization-encrypted orbital angular momentum multiplexed metasurface holography. ACS Nano. 2020;14:5553-9.

87. Lim KT, Liu H, Liu Y, Yang JK. Holographic colour prints for enhanced optical security by combined phase and amplitude control. Nat Commun. 2019;10:25.

88. Wei Q, Sain B, Wang Y, Reineke B, Li X, Huang L, Zentgraf T. Simultaneous spectral and spatial modulation for color printing and holography using all-dielectric metasurfaces. Nano Lett. 2019;19:8964-71.

89. Bao Y, Yu Y, Xu H, Guo C, Li J, Sun S, Zhou Z-K, Qiu C-W, Wang X-H. Full-colour nanoprint-hologram synchronous metasurface with arbitrary hue-saturation-brightness control. Light Sci Appl. 2019;8:95.

90. Hu Y, Luo X, Chen Y, Liu Q, Li X, Wang Y, Liu N, Duan H. 3D-integrated metasurfaces for full-colour holography. Light Sci Appl. 2019:8:86.

91. Li L, Cui TJ, Ji W, Liu S, Ding J, Wan X, Li YB, Jiang M, Qiu C-W, Zhang S. Electromagnetic reprogrammable codingmetasurface holograms. Nat Commun. 2017;8:197.

92. Zhang X, Jiang W, Jiang H, Wang Q, Tian H, Bai L, Luo Z, Sun S, Luo Y, Qiu C-W. An optically driven digital metasurface for programming electromagnetic functions. Nat Electron. 2020;3:165-71.

93. Zhang L, Chen X, Liu S, Zhang Q, Zhao J, Dai J, Bai G, Wan X, Cheng Q, Castaldi G. Space-time-coding digital metasurfaces. Nat Commun. 2018:9:4334.

94. Zhang M, Pu M, Zhang F, Guo Y, He Q, Ma X, Huang Y, Li X, Yu H, Luo X. Plasmonic metasurfaces for switchable photonic spin-orbit interactions based on phase change materials. Adv Sci. 2018;5:1800835.

95. Malek SC, Ee H-S, Agarwal R. Strain multiplexed metasurface holograms on a stretchable substrate. Nano Lett. 2017;17: $3641-5$.

96. Li J, Kamin S, Zheng G, Neubrech F, Zhang S, Liu N. Addressable metasurfaces for dynamic holography and optical information encryption. Sci Adv. 2018;4:eaar6768.

97. Wuttig M, Yamada N. Phase-change materials for rewriteable data storage. Nat Mater. 2007;6:824-32.

98. Qu Y, Li Q, Du K, Cai L, Lu J, Qiu M. Dynamic thermal emission control based on ultrathin plasmonic metamaterials including phase-changing material GST. Laser Photonics Rev. 2017;11:1700091.

99. Raeis-Hosseini N, Rho J. Metasurfaces based on phase-change material as a reconfigurable platform for multifunctional devices. Materials. 2017;10:1046.

100. Gao Y, Fan Y, Wang Y, Yang W, Song Q, Xiao S. Nonlinear holographic all-dielectric metasurfaces. Nano Lett. 2018;18: 8054-61.

101. Reineke B, Sain B, Zhao R, Carletti L, Liu B, Huang L, De Angelis C, Zentgraf T. Silicon metasurfaces for third harmonic geometric phase manipulation and multiplexed holography. Nano Lett. 2019;19:6585-91.

102. Schlickriede C, Waterman N, Reineke B, Georgi P, Li G, Zhang S, Zentgraf T. Imaging through nonlinear metalens using second harmonic generation. Adv Mater. 2018;30:1703843.

103. Schlickriede C, Kruk SS, Wang L, Sain B, Kivshar Y, Zentgraf T. Nonlinear imaging with all-dielectric metasurfaces. Nano Lett. 2020;20:4370-6.

104. Wang L, Kruk S, Koshelev K, Kravchenko I, Luther-Davies B, Kivshar Y. Nonlinear wavefront control with all-dielectric metasurfaces. Nano Lett. 2018;18:3978-84.

105. Li G, Wu L, Li KF, Chen S, Schlickriede C, Xu Z, Huang S, Li W, Liu Y, Pun EY. Nonlinear metasurface for simultaneous control of spin and orbital angular momentum in second harmonic generation. Nano Lett. 2017;17:7974-9.

106. Hu G, Hong X, Wang K, Wu J, Xu H-X, Zhao W, Liu W, Zhang S, Garcia-Vidal F, Wang B. Coherent steering of nonlinear chiral valley photons with a synthetic au-WS 2 metasurface. Nat Photonics. 2019;13:467-72.

107. Almeida E, Bitton O, Prior Y. Nonlinear metamaterials for holography. Nat Commun. 2016;7:12533.

108. Lin Z, Huang L, Xu ZT, Li X, Zentgraf T, Wang Y. Four-wave mixing holographic multiplexing based on nonlinear metasurfaces. Adv Opt Mater. 2019;7:1900782. 
109. Hong X, Hu G, Zhao W, Wang K, Sun S, Zhu R, Wu J, Liu W, Loh KP, Wee ATS. Structuring nonlinear wavefront emitted from monolayer transition-metal dichalcogenides. Research. 2020;2020:9085782.

110. Chen Y, Yang X, Gao J. 3D Janus plasmonic helical nanoapertures for polarization-encrypted data storage. Light Sci Appl. 2019;8:45.

111. Chen K, Ding G, Hu G, Jin Z, Zhao J, Feng Y, Jiang T, Alù A, Qiu CW. Directional janus metasurface. Adv Mater. 2019;32: 1906352

112. Frese $D$, Wei $Q$, Wang $Y$, Huang L, Zentgraf T. Nonreciprocal asymmetric polarization encryption by layered plasmonic metasurfaces. Nano Lett. 2019;19:3976-80.

113. Zhou Y, Kravchenko II, Wang H, Zheng H, Gu G, Valentine J. Multifunctional metaoptics based on bilayer metasurfaces. Light Sci Appl. 2019;8:80.

114. Molesky S, Lin Z, Piggott AY, Jin W, Vucković J, Rodriguez AW. Inverse design in nanophotonics. Nat Photonics. 2018;12: 659-70.

115. Lin Z, Groever B, Capasso F, Rodriguez AW, Lončar M. Topology-optimized multilayered metaoptics. Phys Rev Appl. 2018;9:044030

116. Ma W, Cheng F, Liu Y. Deep-learning-enabled on-demand design of chiral metamaterials. ACS Nano. 2018;12:6326-34.

117. Liu Z, Zhu D, Rodrigues SP, Lee K-T, Cai W. Generative model for the inverse design of metasurfaces. Nano Lett. 2018;18: 6570-6.

118. Gao H, Wang Y, Fan X, Jiao B, Li T, Shang C, Zeng C, Deng L, Xiong W, Xia J. Dynamic 3D meta-holography in visible range with large frame number and high frame rate. Sci Adv. 2020;6:eaba8595.

119. Faraji-Dana M, Arbabi E, Arbabi A, Kamali SM, Kwon H, Faraon A. Compact folded metasurface spectrometer. Nat Commun. 2018:9:4196.

120. Rubin NA, D'Aversa G, Chevalier P, Shi Z, Chen WT, Capasso F. Matrix fourier optics enables a compact full-stokes polarization camera. Science. 2019;365:eaax1839.

121. Kwon H, Arbabi E, Kamali SM, Faraji-Dana M, Faraon A. Single-shot quantitative phase gradient microscopy using a system of multifunctional metasurfaces. Nat Photonics. 2020;14:109-14.

122. Zhu L, Liu X, Sain B, Wang M, Schlickriede C, Tang Y, Deng J, Li K, Yang J, Holynski M. A dielectric metasurface optical chip for the generation of cold atoms. Sci Adv. 2020;6:eabb6667.

\section{Publisher's Note}

Springer Nature remains neutral with regard to jurisdictional claims in published maps and institutional affiliations.

\section{Submit your manuscript to a SpringerOpen ${ }^{\circ}$ journal and benefit from:}

- Convenient online submission

Rigorous peer review

- Open access: articles freely available online

- High visibility within the field

- Retaining the copyright to your article

Submit your next manuscript at $\boldsymbol{\nabla}$ springeropen.com 